\title{
A Geometric Characterization of Normal Two-Dimensional Singularities of Multiplicity Two with $p_{a} \leqq 1$
}

By

\author{
Masataka TOMARI*
}

\section{Introduction}

A normal two-dimensional singularity $(V, p)$ is a germ of normal two-dimensional complex analytic space $V$ with a reference point $p$. Let $(V, p) \stackrel{\psi}{\longleftarrow}(\tilde{V}, A)$ be a resolution of $(V, p)$ with exceptional set $A$. The geometric genus of a normal two-dimensional singularity ( $V$, $p)$ is the integer $p_{g}(V, p)$ defined by

$$
p_{g}(V, p)=\operatorname{dim}_{c}\left(R^{1} \psi_{*} O_{\tilde{v}}\right)_{p}
$$

The arithmetic genus of a normal two-dimensional singularity ( $V$, $p)$ is the integer $p_{a}(V, p)$ defined by

$$
p_{a}(V, p)=\sup _{\substack{D>0 \\|D| \subset A}} p_{a}(D)
$$

Here, the integer $p_{a}(D)$ is the virtual genus of the divisor $D$ on $\tilde{V}$. These two integers are independent of the choice of the resolutions (see [11] [18]). See [3] [4] [10] [11] [12] [13] [17] for the basic facts on two-dimensional singularities and those numerical invariants.

The condition $p_{g}=0$ is equivalent to the condition $p_{a}=0$ (Artin [2] [3]). For the singularity of multiplicity two, it satisfies the condition $p_{g}=0$ if and only if it can be resolved by a succession of blowing-ups at point (i. e., it is an absolutely isolated singularity) (Satz 1. of Brieskorn [4]).

The goal in this paper is a characterization of the normal two-

Communicated by S. Nakano, November 24, 1981. Revised November 28, 1982.

* Research Institute for Mathematical Sciences, Kyoto University, Kyoto 606, Japan. 
dimensional singularity of multiplicity two with $p_{a} \leqq 1$ by means of a special resolution below (see also §1).

Let $(V, p)$ be a normal two-dimensional singularity. A resolution of the singularity $(V, p)$ is obtained by the following process (Zariski [22]);

$$
\begin{aligned}
& \sigma_{1} ; V_{1} \longrightarrow V \text { the blowing up of } V \text { at } p, \\
& T_{1} ; \widetilde{V}_{1} \longrightarrow V_{1} \text { the normalization of } V_{1} \\
& \sigma_{2} ; V_{2} \longrightarrow \widetilde{V}_{1} \text { the blowing up of } \widetilde{V}_{1} \text { at a point in the singular } \\
& T_{2} ; \widetilde{V}_{2} \longrightarrow V_{2} \text { the normalization of } V_{2} \text {, and so on. }
\end{aligned}
$$

Moreover, this process ends in finite steps.

The result of this paper is the following.

Theorem. Let $(V, p)$ be a normal two-dimensional singularity of multiplicity two. The condition $p_{a} \leqq 1$ is satisfied if and only if the normalization $T_{i}$ is trivial or is obtained by a blowing up of $V_{i}$ along a non-singular rational curve in the singular locus of $V_{i}, 1 \leqq i$.

A normal singularity $(V, p)$ of multiplicity two is a hypersurface (see [1] [5]). It can be represented as follows.

$$
(V, p)=\left(\left\{(x, y, z) \in U \mid z^{2}-g(x, y)=0\right\},(0,0,0)\right) .
$$

Here, the set $U$ is an open neighborhood of the origin $o=(0,0,0)$ in $\boldsymbol{C}^{3}$, the function $g(x, y)$ is a convergent power series at $(0,0)$. The defining equation of the normal two-dimensional singularity of multiplicity two, up to the equisingular class of the curve $\{g(x, y)$ $=0\}, o$, can be obtained for the given dual gragh of the exceptional set (Tjurina [17] for the case $p_{a}=0$, see Laufer [13], in general). The theorem can be checked after the classifications in the both class above. The classification of dual graghs of the exceptional sets in the minimal resolutions of the singularities of multiplicity two with $p_{a} \leqq 1$ is done (Artin [3] for the case with $p_{a}=0$, Wagreich [18], Yau [21] for the case with $\left.p_{a}=1\right)$.

The proof of the theorem in this paper is not based on the classification as above, but is based on the explicit computations in the reduction of the singularity to the absolutely isolated singularities 
(in the proof of Lemma 5).

In $\S 1$, the construction of a sequence of modifications, which plays an important role in this paper, is given. In $\S 2$, the proof of the theorem is given. In $\S 3$, the computation which is essential in this paper is done. In $\S 4$, three remarks are given.

The author gives his thanks to Lê Dũng Tráng, A. Fujiki, Kimio Watanabe, I. Naruki, and K. Saito for many suggestions and encouragements.

\section{§1. A Canonical Resolution for the Normal Two-Dimensional Singularity of Multiplicity Two}

(1.1) In this section, for a normal two-dimensional singularity of multiplicity two, a resolution by a sequence of modifications is constructed, which plays an important role in this paper. Some preliminary remarks on the resolution are given. This method is rather standard (e. g., Kirby [9], Horikawa [8], Laufer [13]), and is useful for the study to see how the singularity becomes the absolutely isolated singularities, step by step.

(1.2) Let $(V, p)$ be a normal two-dimensional singularity of multiplicity two represented as $\left\{(x, y, z) \in U \mid z^{2}-g(x, y)=0\right\}$ (cf. Introduction). After the blowing up of $V$ at $p$, the following commutative diagram follows.

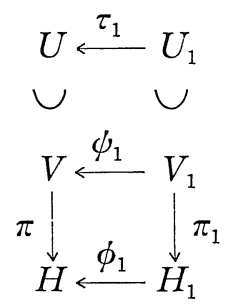

Here, the analytic space $H$ is the hyperplane in $C^{3}$ defined by $\{z=0\}$, the map $\pi$ is the restriction to $V$ of the projection from $\mathbb{C}^{3}$ to $H ;(x, y, z) \longrightarrow(x, y, 0)$.

The map $\tau_{1}$ is the blowing up of $U$ at $p$, the analytic space $U_{1}$ 
(resp. the analytic space $V_{1}$, resp. the analytic space $H_{1}$ ) is the strict transformation of $U$ (resp. $V$, resp. $H$ ) by the blowing up $\tau_{1}$. The map $\phi_{1}$ (resp. the map $\phi_{1}$ ) is the restriction to $V_{1}$ (resp. $H_{1}$ ) of the map $\tau_{1}$. The existence of the map $\pi_{1}$ which commutes the above diagram follows from the transversality of the map $\pi$ (i. e., the multiplicity of $V$ at $p=$ the degree of the map $\pi$ ).

The singular locus of $V_{1}$ is either a non-singular rational curve or a point (when mult $g \leqq 3$ ), since the multiplicity of $V$ is equal to two.

Let the integer $m$ be the multiplicity of the analytic space $\{g(x$, $y)=0\}$ at $(0,0)$ in $H$. After the succession of $\left[\frac{m}{2}\right]-1$ blowingups of $V_{1}$, say $\tau_{2}, \ldots, \tau_{\left[\frac{m}{2}\right]}$, along the non-singular rational curves which are contained in the singular locus, the normalization of $V_{1}$, $V_{\left[\frac{m}{2}\right]} \longrightarrow V_{1}$ follows in the commutative diagram,

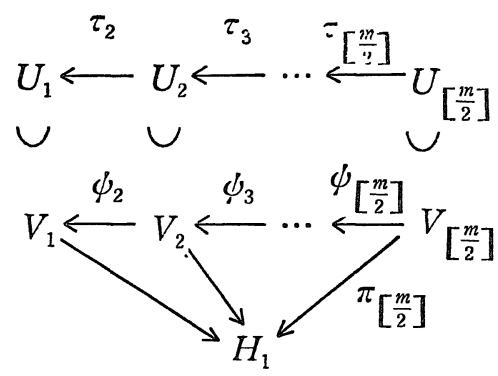

Here, the analytic space $U_{i}$ (resp. the analytic space $V_{i}$ ) is the strict transformation of $U_{i-1}$ (resp. $V_{i-1}$ ) by the blowing-up $\tau_{i}$. The map $\phi_{i}$ is the restriction of the blowing-up $\tau_{i}$ to $V_{i}$. The map $\pi_{i}$ is the composition of the map $\pi_{1} \circ \psi_{2} \circ \ldots \circ \psi_{i}: V_{i} \longrightarrow H_{1}, 2 \leqq i \leqq\left[\frac{m}{2}\right]$. The integer $\left[\frac{m}{2}\right]-1$ is denoted by the integer $\gamma_{1}$ in this paper.

The singular locus of the analytic space $V_{1+r_{1}}$ equals to the singular locus of the analytic space $H_{1} \cap V_{1+r_{1}}$. Here, the notation $H_{1}$ is also used for the strict transformation of the analytic space $H_{1}$ by the blowing-up $\tau_{2} \circ \tau_{3} \circ \ldots \circ \tau_{\left[\frac{m}{2}\right]}$ which induces the isomorphism on $H_{1}$.

The sequence of the modifications $V_{i}$ of $V$, mentioned in the introduction, is defined as the sequence of the strict transforms of $V$ 
in a sequence of blowing-ups of $V \cap H$ in $H$ with the points $\left\{p_{k}\right\}$ as center in the following way.

If $V_{1+r_{1}}$ is non-singular, we stop the construction of the sequence of modifications of $V$. If $V_{1+\gamma_{1}}$ has singularity, we choose a point of $V_{1+r_{1}}$, say $p_{2}$, where $V_{1+r_{1}}$ is singular. We define the integers $T(1)$ and $T(2)$ by the equalities $T(1)=1$ and $T(2)=T(1)+\gamma_{1}+1$. The sequence of modifications of $V_{T(2)-1}$, which consists of the data $\left(U_{T(2)+j}, V_{T(2)+j}, H_{2}, \tau_{T(2)+j}, \psi_{T(2)+j}, \pi_{T(2)+j}, \dot{\phi}_{2}\right) 0 \leqq j \leqq \gamma_{2}$, is defined for the data $\left(U_{T(2)-1}, V_{T(2)-1}, H_{1}, \pi_{T(2)-1}, p_{2}\right)$ by the same procedure in the construction of the sequence of the modifications of $V$ which consists of the data $\left(U_{1+j}, V_{1+j}, H_{2}, \tau_{1+j}, \phi_{1+j}, \pi_{1+j}, \phi_{1}\right) 0 \leqq j \leqq \gamma_{1}$ associated to the data $(U, V, H, \pi, p)$. Here, the integer $\gamma_{2}$ is defined by $\left[\frac{m_{2}}{2}\right]-1$, where the integer $m_{2}$ is the multiplicity of the reduced analytic space $V_{T(2)-1} \cap H_{1}$ at $p_{2}$.

Continuing this procedure, we have the following data.

The strictly increasing sequence of integers $\{T(k)\} \quad 1 \leqq k$, is determined by the equalities $T(1)=1$ and $T(k)=T(k-1)+\gamma_{k-1}+1$ for $2 \leqq$ $k$, where the non-negative integers $\left\{\gamma_{k}\right\} \quad 1 \leqq k$ are defined as follows.

The sequence of modifications of $V_{T(k)-1}$, which consists of the data $\left(U_{T(k)+j}, V_{T(k)+j}, H_{k}, \tau_{T(k)+j}, \phi_{T(k)+j}, \pi_{T(k)+j}, \phi_{k}\right) 0 \leqq j \leqq \gamma_{k}$, is defined for the data $\left(U_{T(k)-1}, V_{T(k)-1}, H_{k-1}, \pi_{T(k)-1}, p_{k}\right)$ by the same procedure in the construction of the sequence of modifications of $V$ which consists of the data $\left(U_{1+j}, V_{1+j}, H_{1}, \tau_{1+j}, \phi_{1+j}, \pi_{1+j}, \phi_{1}\right) 0 \leqq j \leqq \gamma_{1}$ associated to the data $(U, V, H, \pi, p)$. Here, the integer $\gamma_{k}$ is defined by $\left[\frac{m_{k}}{2}\right]-1$, where the integer $m_{k}$ is the multiplicity of the reduced analytic space $V_{T(k)-1} \cap H_{k-1}$ at $p_{k}$.

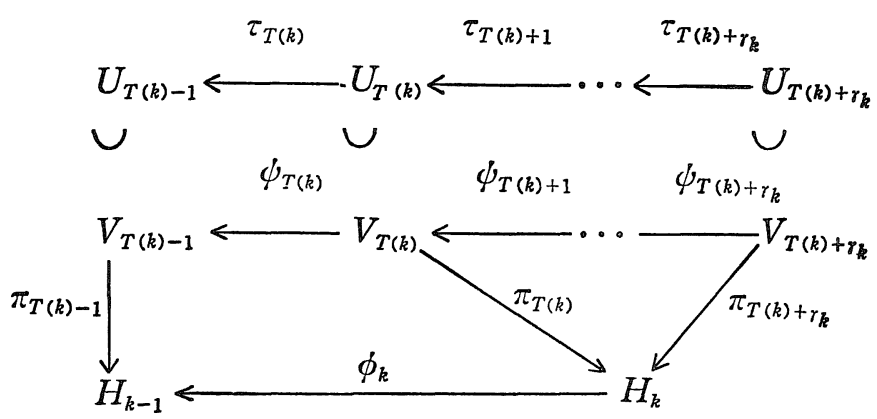


The sequence of modifications of $V$ as above can reach to the canonical resolution of $(V, p)$ which is noted in Introduction.

(1.3) Especially, if $\left\{p_{k}\right\} \quad 1 \leqq k \leqq k_{0}$ contains the set of all singular points of the strict transformations of $V \cap H$, then all the singular points of the analytic space $V_{T\left(k_{0}\right)+\gamma_{k}}$ are absolutely isolated, since the singularity of analytic space $H_{k_{0}} \cap V_{T\left(k_{0}\right)+r_{k_{0}}}$ is one of the following types,

(i) of multiplicity two,

(ii) of multiplicity three with at least two distinct tangents.

(1.4) Some preliminaries are stated here.

Let $(V, p)$ be a normal two-dimensional singularity, and $(V, p)$ $\stackrel{\psi}{\longleftarrow}(\widetilde{V}, A)$ be a resolution of $(V, p)$ with the decomposition into the irreducible components of the exceptional set $A=\cup_{j=1}^{w} A_{j}$. Each analytic set $A_{j}$ determines a valuation $v_{j}$ on the quotient field of the local domain $O_{V, p}$ with the maximal ideal $m_{V, p}$ by $; v_{j}(f)=$ the vanishing order cf $\phi^{*} f$ on $A_{j}$ for $f \in O_{V, p}, 1 \leqq j \leqq w$.

The maximal ideal cycle for the blowing down $\psi$ is the divisor $Y_{\psi}$ on $\widetilde{V}$ defined by

$$
Y_{\psi}=\sum_{j=1}^{w}\left\{\min _{f \in m_{V, p}} v_{j}(f)\right\} A_{j} .
$$

In general, we have an element $h \in m_{V, p}$, such that

$$
Y_{\psi}=\sum_{j=1}^{w} v_{j}(h) A_{j} \text {. }
$$

One can check this by noting Proposition 2. 12 [19].

Hence we have the following equality.

$$
Y_{\psi}=\min _{f \in m_{V, p}}\left\{\sum_{j=1}^{w} v_{j}(f) A_{j}\right\} .
$$

If the inverse image of maximal ideal $\phi^{-1}\left(m_{V, p}\right)$ is locally principal in $O_{\tilde{v}}$, the equality

$$
-\left(Y_{\psi}\right)^{2}=\text { the multiplicity of } V \text { at } p
$$

holds (Theorem 2.7 of Wagreich [18]).

Moreover, assume that the multiplicity of $V$ at $p$ is two and that $(V, p) \stackrel{\psi}{\longleftarrow}(\widetilde{V}, A)$ is the resolution of $(V, p)$ constructed as in (1.2) 
with the diagram

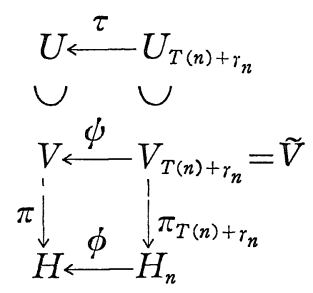

The set of divisors $\left\{E_{i}^{(j)}\right\} i \leqq j$ on $H_{j}, 1 \leqq j \leqq n$, are defined inductively as follows,

$E_{j}^{(j)}=$ the exceptional set of the blowing-up $\phi_{j}$ in $H_{j}$,

$E_{i}^{(j)}=$ the strict transformation of $E_{i}^{(i)}$ by the sequence of modifications above in $H_{j}, i<j$.

The line bundle defined by a divisor $E$ is denoted by $[E]$. Applying the computation in p. 84 [4] to our special resolution, we have the following formula on the canonical line bundles.

$$
K_{\tilde{V}}=i^{*}\left(\tau^{*}\left(K_{U}\right)\right) \otimes\left[-\sum_{i=1}^{n} \gamma_{i} Y_{i}\right]
$$

Here, $K_{\check{V}}\left(\operatorname{resp} . K_{U}\right)$ is the canonical line bundle of $\tilde{V}$ (resp. $U$ ), the divisor $Y_{i}$ on $\tilde{V}$ is the maximal ideal cycle for the resolution of the singularity $\left(V_{T(i)-1}, p_{i}\right)$ in the sequence of modifications above, $1 \leqq i \leqq n$, and the map $i ; V_{T(n)+r_{n}} \longrightarrow U_{T(n)+r_{n}}$ is the inclusion map.

Let the divisor $\phi^{-1}\left(E_{i}^{(i)}\right)$ on $H_{n}$ be the total transformation of the divisor $E_{i}^{(i)}$ on $H_{i}$ by the sequence of modifications above, $1 \leqq i \leqq n$. Representing the situation explicitly with the defining equation, one can show the following equality.

$$
\pi_{T(n)+r_{n}}^{-1}\left(\phi^{-1}\left(E_{i}^{(i)}\right)\right)=Y_{i} \text { for } 1 \leqq i \leqq n 。
$$

Hence, the canonical divisor on $\tilde{V}$ whose support is contained in $A$, which is also denoted by $K_{\tilde{V}}$, is written as follows.

$$
\begin{aligned}
K_{\tilde{V}} & =-\sum_{i=1}^{n} \gamma_{i} Y_{i} \\
& =\pi_{T(n)+\gamma_{n}}^{-1}\left(-\sum_{i=1}^{n} \gamma_{i} \phi^{-1}\left(E_{\imath}^{(i)}\right)\right) .
\end{aligned}
$$

Moreover, the equalities 
$(1.4 .6)$

$$
\begin{aligned}
& \left(Y_{i}\right)^{2}=-2 \quad 1 \leqq i \leqq n \quad(\text { from }(1.4 .2)), \\
& Y_{i} \circ Y_{j}=0 \quad i \neq j
\end{aligned}
$$

follow.

\section{§. The Proof of Theorem}

(2.1) The theorem follows from the four lemmas below.

Lemma 1. Let $(V, p)$ be a normal two-dimensional singularity of multiplicity two, and $(\tilde{V}, A) \longrightarrow(V, p)$ be the resolution of $(V, p)$ as in $\S 1$. If the integer $\max _{1 \leqq i \leqq n} \gamma_{i}$ is not zero, the following inequality holds.

$$
p_{a} \geqq \frac{1}{4} \sum_{r_{i}: \text { єven }}\left(\gamma_{i}\right)^{2}+\frac{1}{4} \sum_{\gamma_{i}: \text { odd }}\left\{\left(\gamma_{i}\right)^{2}-1\right\}+1
$$

Proof. Take the maximum of the set of integers

$$
\left\{p_{a}(D) \mid D=\sum_{i=1}^{n} \alpha_{i} Y_{i}, \alpha_{i} \in Z, \alpha_{i} \geqq 0,1 \leqq i \leqq n, D \neq 0\right\} .
$$

This integer coincides with the right hand side of the above inequality. We can check this by using the equalities (1.4.4) and (1.4.6). The assertion follows from the definition of $p_{a}$. Q. E. D.

The next lemma is due to E. Horikawa (Lemma 6 of [8]).

Lemma 2. Let $(V, p)$ be a normal two-dimensional singularity of multiplicity two. The following equality holds.

$$
p_{g}=\frac{1}{2} \sum_{i=1}^{n} \gamma_{i}\left(\gamma_{i}+1\right) \text {. }
$$

Proof. The representation of $(V, p) ;\left\{z^{2}-g(x, y)=0\right\}$ can be taken with a polynomial $g([15])$. There exists a double plane $S$ on which the singularity $(V, p)$ lies. Let $S \stackrel{\psi}{\longleftarrow} \tilde{S}$ be a resolution of the singularities of $S$. Using Leray spectral sequence, the equality $\chi\left(O_{\tilde{s}}\right)-\chi\left(O_{S}\right)=-\operatorname{dim}_{c}\left(R^{1} \psi_{*} O_{\tilde{s}}\right)+\operatorname{dim}_{c}\left(R^{2} \psi_{*} O_{\tilde{s}}\right)$ follows. On the other hand, the equality $R^{2} \psi_{*} O_{\tilde{s}}=0$ ([16]) holds. The assertion follows from Lemma 6 of [8].

Q. E. D. 
Before stating the remaining lemmas, an invariant for a normal two-dimensional Gorenstein singularity $(V, p)$ (Hence, it possesses a nowhere vanishing holomorphic two-form, say $\omega$, on $V-\{p\}$. See Theorem 1.6 of [6]) is introduced.

Let $(V, p) \stackrel{\psi}{\longleftarrow}(\tilde{V}, A)$ be a resolution of the normal two-dimensional Gorenstein singularity $(V, p)$ with the decomposition into the irreducible components of the exceptional set $A=\bigcup_{j=1}^{w} A_{j}$. The number $L(V, p)$ for $(V, p)$ is defined via $\psi$ by

$$
L(V, p)=\min \left\{\alpha \in Z \mid-K_{\tilde{v}} \leqq \alpha Y_{\psi}\right\} .
$$

Proposition 3. Let the situation be as above. The number $L(V$, $p$ ) is non-negative and is independent of the choice of resolutions of the siugularity $(V, p)$.

Proof. We fix a resolution of $(V, p)$ as above. There is an index $i$ in the set $\{1, \ldots, w\}$ such that the coefficient of $-K_{\tilde{V}}$ on $A_{i}$ is non-negative (Proposition 2.1 of [12] and Lemma 3.1 of [19]). Hence, the inequality $L \geqq 0$ is clear, for every fixed resolution of ( $V$, p).

We shall show the equality $L_{V^{\prime}}=L_{\tilde{V}}$ in the following situation.

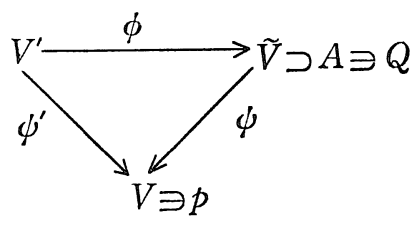

Here $(V, p) \stackrel{\psi}{\longleftarrow}(\tilde{V}, A)$ is a resolution of $(V, p)$ as above. The map $\phi: V^{\prime} \longrightarrow \widetilde{V}$ is the blowing up of $\tilde{V}$ at a point $Q$ in $A$, and the map $\psi^{\prime}:\left(V^{\prime}, \mid \phi^{-1}(A) !\right) \longrightarrow(V, p)$ is the resolution of $(V, p)$ which is the composition of the maps $\psi$ and $\phi$. The integers $L_{\tilde{V}}$ and $L_{V^{\prime}}$ are defined as follows.

$$
\begin{aligned}
& L_{\tilde{V}}=\min \left\{\alpha \in Z \mid-K_{\tilde{V}} \leqq \alpha Y_{\psi^{\prime}}\right\}, \\
& L_{V^{\prime}}=\min \left\{\alpha \in Z \mid-K_{V^{\prime}} \leqq \alpha Y_{\psi^{\prime}}\right\},
\end{aligned}
$$

where the divisor $K_{V^{\prime}}$ on $V^{\prime}$ is the canonical divisor of $V^{\prime}$ whose supports lie in $\left|\phi^{-1}(p)\right|=\left|\phi^{-1}(A)\right|$. In general, the inequality $\phi^{-1}\left(Y_{\psi}\right)$ 
$\leqq Y_{\psi^{\prime}}$ holds. We have the following inequalities

$$
\begin{aligned}
-K_{V^{\prime}} & \leqq-K_{V^{\prime}}+\left|\phi^{-1}(Q)\right|=\phi^{-1}\left(-K_{\tilde{V}}\right) \quad \text { (Hurwitz formula) } \\
& \left.\leqq L_{\tilde{V}} \phi^{-1}\left(Y_{\psi}\right) \leqq L_{\tilde{V}} Y_{\psi} \text { (Because } L_{\tilde{V}} \geqq 0\right) .
\end{aligned}
$$

Hence, we have the inequality $L_{V^{\prime}} \leqq L_{\tilde{V}}$.

Let the divisor $A_{j}^{\prime}$ on $V^{\prime}$ be the strict transformation of $A_{j}, 1 \leqq j$ $\leqq w$. The coefficient of the divisor $Y_{\psi^{\prime}}\left(\operatorname{resp} . K_{V^{\prime}}\right)$ on $A_{j}^{\prime}$ is equal to the coefficient of the divisor $Y_{\psi}$ (resp. $\left.K_{\tilde{V}}\right), 1 \leqq j \leqq w$. Hence, the inequality $-K_{V^{\prime}} \leqq L_{V^{\prime}} Y_{\psi^{\prime}}$ induces the inequality $-K_{\hat{v}} \leqq L_{V^{\prime}} Y_{\psi^{\prime}}$. We have the inequality $L_{V^{\prime}} \geqq L_{\tilde{V}}$.

By the theorem of Hopf (Theorem 5.7 and Theorem 5.10 of [10]), we have the assertion of Proposition.

Q. E. D.

On the other hand, the geometric genus $p_{g}(V, p)$ is represented as follows (p. 604 Laufer [11]),

$$
p_{g}(V, p)=\operatorname{dim}_{c}\left\{\Gamma\left(\tilde{V}-A, \Omega_{\tilde{V}}^{2}\right) / \Gamma\left(\tilde{V}, \Omega_{\tilde{V}}^{2}\right)\right\} .
$$

Here, the analytic space $V$ is taken to be a Stein space which possesses $p$ as its only singularity, and the sheaf of holomorphic twoforms on $\tilde{V}$ is denoted by $\Omega_{\tilde{V}}^{2}$. In the case of Gorenstein $(V, p)$, the $O_{V, p}$-module $\Gamma\left(\tilde{V}-A, \Omega_{\tilde{V}}^{2}\right) / \Gamma\left(\tilde{V}, \Omega_{\tilde{V}}^{2}\right)$ is principal, and is written as $O_{V, p} \operatorname{cls}[\omega]$.

Proposition 4. Let $(V, p)$ be a normal two-dimensional Gorenstein singula rity. We have the equality

$$
L(V, p)=\max _{h \in m_{V, p}} \operatorname{dim}_{c} \boldsymbol{C}\left[\psi^{*} h\right] \operatorname{cls}[\omega] .
$$

Here, $\boldsymbol{C}\left[\psi^{*} h\right] \operatorname{cls}[\omega]$ is the $\boldsymbol{C}_{\text {-vector subspace of } \Gamma\left(V-A, \Omega_{\vec{V}}^{2}\right) /}$ $\Gamma\left(V, \Omega_{\tilde{V}}^{2}\right)$ corresponding to the $\boldsymbol{C}$-vector subspace $\sum_{1} \boldsymbol{C}\left(\left(\psi^{*} h\right)^{1} \omega\right)$ in $\Gamma\left(\widetilde{V}-A, \Omega_{\tilde{V}}^{2}\right)$.

Proof. Let us take $h \in m_{V, p}$. The holomorphic two-form $\omega$ on $\tilde{V}-A$ is extended to the meromorphic two-form on $\tilde{V}$ whose pole divisor is equal to $K_{\tilde{v}}$. Hence, we have the equality

$$
\operatorname{dim}_{\boldsymbol{c}} \boldsymbol{C}\left[\psi^{*} h\right] \operatorname{cls}[\omega]=\min \left\{\alpha \in \boldsymbol{Z} \mid K_{\tilde{\nu}}+\alpha \sum_{j=1}^{w} v_{j}(h) A_{j} \geqq 0\right\} .
$$

The assertion follows from the equality (1.4.1). Q. E. D. 
Lemma 5. Let $(V, p)$ be a normal two-dimensional singularity of multiplicity two represented as $\left\{(x, y, z) \in U \mid z^{2}-g(x, y)=0\right\} \quad$ (cf. Introduction). If the multiplicity of $\{g(x, y)=0\}$ at $(0,0)$ is less or equal than 5, then the equality $L=\sum_{i=1}^{n} \gamma_{i}$ holds.

A proof of Lemma 5 is given in $\S 3$.

Noting Proposition 4, a theorem due to S. S.-T. Yau (Theorem 3.2 of [20]) can be stated in the following form.

Lemma 6. Let $(V, p)$ be a normal two-dimensional Gorenstein singularity. If the equality $L=p_{g}$ holds, the arithmetic genus $p_{a} \leqq 1$.

Proof of the theorem. If the inequalities $\gamma_{i} \leqq 1,1 \leqq i \leqq n$, hold, the condition of Lemma 5 is satisfied by the definition of the integer $\gamma_{1}$. With Lemma 2 and Lemma 5, the equalities $p_{g}=\frac{1}{2} \sum_{i=1}^{n} \gamma_{i}\left(\gamma_{i}+1\right)=\sum_{i=1}^{n} \gamma_{i}$ $=L$ hold. By Lemma 6, the inequality $p_{a} \leqq 1$ hold. The converse follows from Lemma 1.

Q. E. D.

(2. 2) Remark. In the proof of Lemma 6, the following result (Wagreich [18], also see Corollary 4.2 of [12], the appendix of the text [7]) is used

Let $(V, p) \stackrel{\psi}{\longleftarrow}(\tilde{V}, A)$ be a resolution of a normal two-dimensional singularity $(V, p)$. Let the divisor $Z_{0}$ on $\tilde{V}$ be the fundamental divisor of Artin for the blowing down $\psi[3]$.

Theorem 7 (cf. Remark (4.3)). The condition $p_{a}=1$ is equivalent to the condition $p_{a}\left(Z_{0}\right)=1$.

\section{§3. The Proof of Lemma 5}

(3.1) we shall show that the problem is the existence of the irreducible component of the reduced analytic space $H \cap V$ with the special numerical conditions (in the Assertion (3.2)).

Let $(V, p)$ be a normal two-dimensional singularity of multiplicity two represented as $\left\{(x, y, z) \in U \mid z^{2}-g(x, y)=0\right\}$ (cf. Introduction). 
By the equalities (1.4.3) and (1.4.5), the number $L$ is written via the resolution which is constructed in (1.2) as follows,

$$
L=\min \left\{\alpha \in Z \mid \sum_{i=1}^{n} \gamma_{i} \phi^{-1}\left(E_{i}^{(i)}\right) \leqq \alpha \phi^{-1}\left(E_{1}^{(1)}\right)\right\} .
$$

The divisor $\phi^{-1}\left(E_{i}^{(i)}\right)$ on $H_{n}$ is written as follows,

$$
\phi^{-1}\left(E_{i}^{(i)}\right)=\sum_{k=1}^{n} s_{k}^{(i)} E_{k}^{(n)}, 1 \leqq i \leqq n \text {, on } H_{n} \text {. }
$$

Here, the set of numbers $\left\{s_{k}^{(i)}\right\} 1 \leqq i, k \leqq n$ are defined inductively by (3. 1. 1)

$$
s_{k}^{(i)}=0, k<i, s_{i}^{(i)}=1 \text {, and } s_{k}^{(i)}=\sum_{j \in \lambda_{k}} s_{j}^{(i)}, k>i,
$$

where the index sets $\lambda_{k}, 2 \leqq k \leqq n$, are defined by

$$
\lambda_{k}=\left\{j \in\{1, \ldots, n-1\} \mid p_{k} \in E_{j}^{(k-1)} \text {, in } H_{k-1}\right\} .
$$

We shall introduce the set of integers $\left\{l_{k}\right\} \quad 1 \leqq k \leqq n$ as follows

$$
l_{k}=\min \left\{\alpha \in \mathbb{Z} \mid \sum_{i=1}^{n} \gamma_{i} s_{k}^{(i)} \leqq \alpha s_{k}^{(1)}\right\} .
$$

We can prove the following equality.

$$
L=\max _{1 \leqq k \leqq n} l_{k} \text {. }
$$

If the equality $\gamma_{a}=0$ holds for some index $a$ in $\{2, \ldots, n\}$ in the resolution constructed in (1.2), the inequality $l_{a} \leqq \max _{j \in \lambda_{a}} l_{j}$ follows from the rule (3.1.1) above.

Hence, to compare $L$ with $\sum_{i=1}^{n} \gamma_{i}$, it is sufficient to study the reduction procedures from the singularity $(V, p)$ to the absolutely isolated singularities in the resolution which is constructed in (1.2).

Let us take an irreducible component $C$ of the reduced analytic space $H \cap V$, and fix it in the rest of (3.1).

Choose all the singular points of the strict transformations of $H \cap$ $V$ by the resolution which is constructed in (1.2) which lie on the strict transformations of $C$, say $q_{1}, \ldots, q_{r}$. We may assume the conditions $p_{i}=q_{i}, 1 \leqq i \leqq r$, in the construction of the sequence of modifications (1.2). The diagram

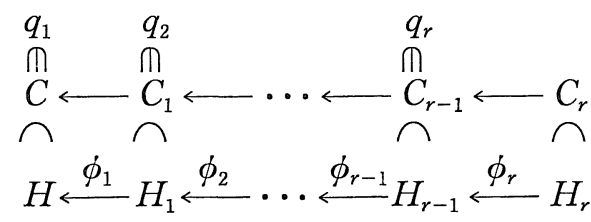


follows, where the strict transform of $C$ in $H_{i}$ by the sequence of modifications above is denoted by $C_{i}, 1 \leqq i \leqq r$.

Let the integer $e_{i}$ be the multiplicity of the analytic space $C^{i}{ }_{-1}$ at $q_{i}, 1 \leqq i \leqq r$. Assume that

$$
e_{1}=e_{2}=\ldots=e_{k_{1}}>e_{k_{1}+1}, \quad\left(\text { or } e_{i}=1,1 \leqq i \leqq r\right) .
$$

About the intersection numbers $E_{i}^{(j)} \circ C_{j}$ in $H_{j}$, we have the equalities

$$
\begin{aligned}
& E_{i}^{(i)} \circ C_{i}=e_{i}, \text { in } H_{i}, 1 \leqq i \leqq r, \\
& E_{i}^{(i+1)} \circ C_{i \perp 1}=e_{i}-e_{i+1}, \text { in } H_{i+1}, 1 \leqq i \leqq r-1 .
\end{aligned}
$$

It follows that $\lambda_{i}=\{i-1\}, 2 \leqq i \leqq k_{1}+1, \lambda_{k_{1}+2}=\left\{k_{1}, k_{1}+1\right\}$. Hence, we have the following equalities,

$$
\begin{aligned}
& l_{i}=\sum_{h=1}^{i} \gamma_{h}, 1 \leqq i \leqq k_{1}+1, \\
& l_{k_{1}+2}=\min \left\{\alpha \in \mathbb{Z} \mid \sum_{h=1}^{k_{1}} \gamma_{h}+\frac{\gamma_{k_{1}+1}+\gamma_{k_{1}+2}}{2} \leqq \alpha\right\} .
\end{aligned}
$$

(3.2) The following assertion is sufficient to prove Lemma 5.

Assertion. There is an irreducible component $C$ of the reduced analytic space $H \cap V$ such that $\gamma_{k_{1}+1}+\gamma_{k_{1}+2} \leqq 1, \gamma_{i}=0, k_{1}+3 \leqq i$, in the terminologies in (3.1) above.

Conventions in (3.2). The germ of the analytic space $D$ at $p$ means $(H \cap V, p)$. The analytic space $(X)_{i}$, which is also denoted by $X_{i}$, for the analytic subspace $X$ of $D$ in $H_{i}$ by the resolution of the singularity $(V, p)$ which is constructed in (1.2). An infinitely near singular point $P$ of the singularity $R$ means a singular point of the strict transformation of $R$ by the blowing-up.

Since the multiplicity of $D$ at $p$ is less than or equal to 5 , by the assumption in Lemma 5, the proof of the assertion is separated into the following four cases.

\section{(i) $D$ : irreducible.}

The sequences of multiplicities of singularities in the resolution tree of $D$ by blowing-ups are classified by noting the relations (3.1. 
2) as follows. The integer $m$ is the multiplicity of $D$ at $p$.

$$
\begin{array}{ll}
m=1, & 1, \\
m=2, & 2, \ldots, 2,1, \\
m=3, & 3, \ldots, 3,1,3, \ldots, 3,2,1 \\
m=4, & 4, \ldots, 4,1,4, \ldots, 4,3,1 \\
& 4, \ldots, 4,2,2, \ldots, 2,1,\left(^{*}\right) \\
m=5, & 5, \ldots, 5,1,5, \ldots, 5,4,1, \\
& 5, \ldots, 5,3,2,1,5, \ldots, 5,2,2,1 .
\end{array}
$$

We must check the numerical conditions of the assertion for each subcase above. As a typical example, we shall do for the case " $5, \ldots, 5,3,2,1 "$ in the following way.

Since $D_{k_{1}+2}$ in $H_{k_{1}+2}$ is smooth, the analytic space $V_{T\left(k_{1}+2\right)+r_{k_{1}+2}}$ has, at worst, absolutely isolated singularities (1.3). Hence, we have $\gamma_{i}=$ 0 for $k_{1}+3 \leqq i$.

To compute the integers $\gamma_{k_{1}+1}$ and $\gamma_{k_{1}+2}$, we note the following data (cf. the equalities $(3.1 .2)$ ).

$$
\begin{aligned}
& D_{k_{1}-1} \circ E_{i}^{\left(k_{1}-1\right)}=0 \quad i \leqq k_{1}-2, \quad D_{k_{1}-1} \circ E_{k_{1}-1}^{\left(k_{1}-1\right)}=5, \\
& \operatorname{mult}_{p_{k_{1}}} D_{k_{1}-1}=5, \quad \text { in } H_{k_{1}-1} \cdot \\
& D_{k_{1}} \circ E_{k_{1}-1}^{\left(k_{1}\right)}=0, \quad D_{k_{1}} \circ E_{k_{1}}^{\left(k_{1}\right)}=5, \quad \operatorname{mult}_{p_{k_{1}+1}} D_{k_{1}}=3, \quad \text { in } H_{k_{1}} \circ \\
& D_{k_{1}+1} \circ E_{k_{1}}^{\left(k_{1}+1\right)}=2, \quad D_{k_{1}+1} \circ E_{k_{1}+1}^{\left(k_{1}+1\right)}=3, \\
& \operatorname{mult}_{p_{k_{1}+2}} D_{k_{1}+1}=2, \quad \text { in } H_{k_{1}+1} .
\end{aligned}
$$

There are two cases as follows.

(a) $\quad\left(H_{k_{1}-1} \cap V_{T\left(k_{1}\right)-1}, p_{k_{1}}\right)=\left(E_{k_{1}-1}^{\left(k_{1}-1\right)} \cup D_{k_{1}-1}, p_{k_{1}}\right)$,

$$
\begin{aligned}
& \operatorname{mult}_{p_{k_{1}}} H_{k_{1}-1} \cap V_{T\left(k_{1}\right)-1}=6, \quad \gamma_{k_{1}}=2 . \\
& \left(H_{k_{1}} \cap V_{T\left(k_{1}+1\right)-1}, p_{k_{1}+1}\right)=\left(D_{k_{1}}, p_{k_{1}+1}\right),
\end{aligned}
$$$$
\text { mult }_{p_{k_{1}+1}} H_{k_{1}} \cap V_{T\left(k_{1}+1\right)-1}=3, \quad \gamma_{k_{1}+1}=0 \text {. }
$$$$
\left(H_{k_{1}+1} \cap V_{T\left(k_{1}+2\right)-1}, p_{k_{1}+2}\right)=\left(D_{k_{1}+1} \cup E_{k_{1}+1}^{\left(k_{1}+1\right)}, p_{k_{1}+2}\right) \text {, }
$$$$
\text { mult }_{p_{k_{1}+2}} H_{k_{1}+1} \cap V_{T\left(k_{1}+2\right)-1}=3, \quad \gamma_{k_{1}+2}=0 \text {. }
$$

(b) $\left(H_{k_{1}-1} \cap V_{T\left(k_{1}\right)-1}, p_{k_{1}}\right)=\left(D_{k_{1}-1}, p_{k_{1}}\right)$, 


$$
\begin{aligned}
& \text { mult }_{p_{k_{1}}} H_{k_{1}-1} \cap V_{T\left(k_{1}\right)-1}=5, \quad \gamma_{k_{1}}=1 . \\
& \left(H_{k_{1}} \cap V_{T\left(k_{1}+1\right)-1}, p_{k_{1}+1}\right)=\left(D_{k_{1}} \cup E_{k_{1}}^{\left(k_{1}\right)}, p_{k_{1}+1}\right), \\
& \text { mult }_{p_{k_{1}+1}} H_{k_{1}} \cap V_{T\left(k_{1}+1\right)-1}=4, \quad \gamma_{k_{1}+1}=1 . \\
& \left(H_{k_{1}+1} \cap V_{T\left(k_{1}+2\right)-1}, p_{k_{1}+2}\right)=\left(D_{k_{1}+1} \cup E_{k_{1}}^{\left(k_{1}+1\right)}, p_{k_{1}+2}\right), \\
& \text { mult }_{p_{k_{1}+2}} H_{k_{1}+1} \cap V_{T\left(k_{1}+2\right)-1}=3, \quad \gamma_{k_{1}+2}=0 .
\end{aligned}
$$

By the similar arguments, we can check the assertion for the case (i). The details are left to the reader.

For the reducible $D$, the proof separates into the following three cases.

(ii) $D={ }_{0} C \cup\left(\cup{ }_{a} B\right)$, where the component ${ }_{0} C$ is irreducible of multiplicity $\geqq 3$ at $p$, the component ${ }_{\alpha} B$ is non-singular at $p$, for all $\alpha$.

(iii) $D=\cup{ }_{\alpha} C$, where the component ${ }_{\alpha} C$ is irreducible of multiplicity $\stackrel{\alpha}{\alpha}$ at $p$, for all $\alpha$.

(iv) $D={ }_{1} C \cup{ }_{2} C$, where the component ${ }_{1} C$ is irreducible of multiplicity two at $p$, and the component ${ }_{2} C$ is irreducible of multiplicity 3 at $p$.

For these three cases, we shall choose the irreducible component that satisfies the conditions of the assertion. The computation about the numerical conditions in the assertion for each subcase is similar to that for the case " $5, \ldots, 5,3,2,1 . "$ in (i), and is left to the reader.

(ii) If the component ${ }_{0} C$ is of type (*) in (i), take ${ }_{0} C$ as $C$ in the assertion. Now assume that the component ${ }_{0} C$ is not of type $(*)$. Consider the subsequence of modifications of $(V, p)$ which is associated to the irreducible component ${ }_{0} C$, as which is constructed to $C$ in $(3.1)$.

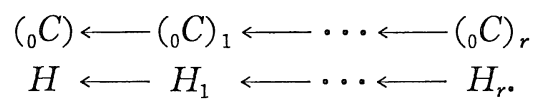

If the condition $\left({ }_{0} C\right)_{r} \cap\left({ }_{\alpha} B\right)_{r}=\dot{\phi}$, for all $\alpha$, are satisfied in $H_{r}$, the 
singularities of $V_{T(r)+r_{r}}$ are absolutely isolated. Or if there is some ${ }_{\alpha} B$ such that the condition $\left({ }_{0} C\right)_{r} \cap\left({ }_{\alpha} B\right)_{r} \neq \phi$ is satisfied in $H_{r}$. After the sequence of modifications of $V_{T(r)+r_{r}}$ associated to the blowing-up $H_{r} \longleftarrow H_{r+1}$ at the point $\left({ }_{0} C\right)_{r} \cap\left({ }_{\alpha} B\right)_{r}$, the singularities of $V_{T(r+1)+r_{r+1}}$ are absolutely isolated. We can choose the ${ }_{\alpha} B$ as $C$ in the assertion.

(iii) Let an infinitely near singular point $Q$ of $D$ of multiplicity $\geqq 3$ be the point such that there is no such a point after $Q$. There is an irreducible component, say ${ }_{0} C$ such that $\left({ }_{0} C\right)_{i}$ contains $Q$ for some $i$, and that the multiplicity of $\left({ }_{0} C\right)_{i}$ at $Q$ is equal to the multiplicity of ${ }_{0} C$ at $p$. Consider the sequence of modifications of the singularity $(V, p)$ which is associated to the irreducible component ${ }_{0} C$, as which is constructed in $(3.1)$.

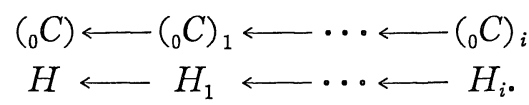

The singularity of $V_{T(i)+r_{i}}$ which is not absolutely isolated is only one point over $Q$. Because if the residual part of $D_{i}$ has a singular point of multiplicity two, the multiplicities of all the irreducible components do not change after these modifications. At most one exceptional curve $E^{(i)}$ in $H_{i}$ transversely meets with each irreducible components of $D_{i}$ at the residual part. (Cf. (1.3) for the case that the residual part of $D_{i}$ is smooth.) Consider the sequence of modifications of $V_{T(i)+r_{i}}$ associated to the blowing-up $H_{i} \longleftarrow H_{i+1}$ at $Q$. If the singularities of $V_{T(i+1)+r_{i+1}}$ are absolutely isolated, we can choose ${ }_{0} C$ as in the assertion.

Or if not, there is an infinitely near singular point, say $Q_{2}$, such that the singularity of $V_{T(i+1)+r_{i+1}}$ over $Q_{2}$ is not absolutely isolated. In this case, the analytic space $D_{i+1}$ is reducible of multiplicity two at $Q_{2}$. These two irreducible components of $\left(D_{i+1}, Q_{2}\right)$, say $\left({ }_{1} C\right)_{i+1}$ and $\left({ }_{2} C\right)_{i+1}$, must have the multiplcity two at $p$ in $H$, and have same tangent in $H_{i}$. They have same multiplicity, say $e(\leqq 2)$, in $H_{i}$. If the multiplicity $e$ is one, the singularities of $V_{T(i+1)+r_{i+1}}$ are absolutely isolated. If the multiplicity $e$ is two, we have the equality $\left(H_{i+1} \cap\right.$ $\left.V_{T(i+1)+r_{i+1}}, Q_{2}\right)=\left(\left({ }_{1} C\right)_{i+1} \cup\left({ }_{2} C\right)_{i+1} \cup E_{i+1}^{(i+1)}, Q_{2}\right)$. We can choose ${ }_{1} C$ (or ${ }_{2} C$ ) as $C$ in the assertion. 
(iv) Consider the sequence of modification of $(V, p)$ associated to the irreducible component ${ }_{2} C$ as in (3.1).

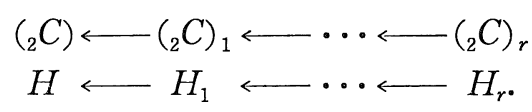

If the condition $\left({ }_{1} C\right)_{r} \cap\left({ }_{2} C\right)_{r}=\phi$ holds in $H_{r}$, the singularities of $V_{T(r)+\gamma_{r}}$ are absolutely isolated. We can choose ${ }_{2} C$ as $C$ in the assertion. In general, suppose that one of the multiplicity of ${ }_{1} C$ and ${ }_{2} C$ drops first in $H_{i}$, the sequences of couples of multiplicity of $\left({ }_{1} C\right)_{j}$ and $\left({ }_{2} C\right)_{j}, 1 \leqq j \leqq r$, in the modifications above are classified as in the following five cases (cf. (i )).

$$
\begin{aligned}
& 1, \quad \ldots \quad i-1, \quad i \text {, } \\
& \text { (1) }(2,3), \ldots,(2,3),(2,2) \text {, } \\
& \text { (2) }(2,3), \ldots,(2,3),(2,1) \text {, } \\
& \text { (3) }(2,3), \ldots,(2,3),(1,3) \text {, } \\
& \text { (4) }(2,3), \ldots,(2,3),(1,2),(1,1) \text {, } \\
& \text { (5) }(2,3), \ldots,(2,3),(1,1) \text {. }
\end{aligned}
$$

In the cases (1) and (3), the condition $\left({ }_{1} C\right)_{i+1} \cap\left({ }_{2} C\right)_{i+1}=\phi$ holds in $H_{i+1}$. We can choose ${ }_{2} C$ as $C$ in the assertion. If the condition $\left({ }_{1} C\right)_{i} \cap\left({ }_{2} C\right)_{i} \neq \phi$ holds in $H_{i}$ in the case (2), after the sequence of modifications of $V_{T(i)+r_{i}}$ associated to the blowing-up $H_{i} \longleftarrow H_{i+1}$ at the point $\left({ }_{1} C\right)_{i} \cap\left({ }_{2} C\right)_{i}$, the condition $\left({ }_{1} C\right)_{i+1} \cap\left({ }_{2} C\right)_{i+1}=\phi$ holds in $H_{i+1}$ o We can choose ${ }_{2} C$ as $C$ in the assertion. If the condition $\left({ }_{1} C\right)_{i+1} \cap$ $\left({ }_{2} C\right)_{i+1} \neq \phi$ holds in $H_{i+1}$ in the case (4), we have the equality $\left({ }_{1} C\right)_{i+1}$ 。 $\left({ }_{2} C\right)_{i+1}=1$.

The multiplicity of $H_{i} \cap V_{T(i)+r_{i}}$ at the point $\left({ }_{1} C\right)_{i+1} \cap\left({ }_{2} C\right)_{i+1}$ is less than or equal to four. We can choose ${ }_{2} C$ as $C$ in the assertion. If the condition $\left({ }_{1} C\right)_{i} \cap\left({ }_{2} C\right)_{i} \neq \phi$ holds in $H_{i}$ in the case (5), we have the equality $\left({ }_{1} C\right)_{i} \circ\left({ }_{2} C\right)_{i}=2$.

The multiplicity of $H_{i} \cap V_{T(i)+r_{i}}$ at the point $\left({ }_{1} C\right)_{i} \cap\left({ }_{2} C\right)_{i}$ is less or equal than three. We can choose ${ }_{2} C$ as $C$ in the assertion.

This completes the proof of Lemma 5 . 


\section{§4. Remarks}

(4.1) Let $(V, p)$ be a normal two-dimensional singularity of multiplicity two. The geometric genus $p_{g}$ is a function of the series of integers $\left\{\gamma_{i}\right\} 1 \leqq i \leqq n$ by Lemma 2. But the arithmetic genus $p_{a}$ is not a function of the series of integers $\left\{\gamma_{i}\right\} 1 \leqq i \leqq n$, in general, as seen in the following example;

$$
\begin{aligned}
& \left\{z^{2}=x^{9}+y^{12}\right\}, o \text { with } p_{a}=4, \\
& \left\{z^{2}=\left(x^{2}+y^{3}\right)^{4}+x^{4} y^{8}\right\}, o \text { with } p_{a}=3,
\end{aligned}
$$

where on the both cases $\gamma_{1}=3, \gamma_{2}=\gamma_{3}=\gamma_{4}=1$.

(4.2) Let $(V, p)$ be a normal two-dimensional singularity embedded in $\left(\boldsymbol{C}^{3}, o\right)$, of multiplicity $\rho$. With the same idea in Lemma 1 , the following proposition follows.

Proposition 8. Let $(V, p)$ be as above. The following inequalities hold.

$$
\begin{aligned}
& p_{a} \geqq \frac{1}{8}(\rho-2)^{2} \rho+1, \quad \text { if } \rho: \text { even } \geqq 4, \\
& p_{a} \geqq \frac{1}{8}(\rho-1)(\rho-3) \rho+1, \quad \text { if } \rho: o d d \geqq 3 .
\end{aligned}
$$

Proof. Let $(V, p) \stackrel{\psi}{\longleftarrow}(\tilde{V}, A)$ be a resolution of $(V, p)$ which is obtained by a succession of blowing-ups with non-singular centers, everywhere in which the multiplicity is constant (Levi-Zariski, see [14]). The canonical divisor $K_{\tilde{V}}$ on $\tilde{V}$ whose support is contained in $A$ is written as follows (see the proof of Satz 1 of [4]),

$$
K_{\tilde{V}}=(2-\rho) Y_{\psi}-Y^{\prime} \text {. }
$$

Here, the divisor $Y_{\psi}$ on $\tilde{V}$ is the maximal ideal cycle for the blowing down $\phi(1.4)$, the divisor $Y^{\prime}$ on $\widetilde{V}$ is effective and is contained in $A$. There is an element $f \in O_{V, p}$ such that the equality $\left(\psi^{*} f\right)-D(f)=Y_{\psi}$ holds, as we remarked in the above of $(1.4 .1)$, where the divisor $\left(\psi^{*} f\right)$ on $\tilde{V}$ is the total transform of the divisor $\{f=0\}$ on $V$, and the divisor $D(f)$ is the strict transform of it. We have the inequality $0 \geqq Y_{\psi^{\circ}} Y^{\prime}=-D(f) \circ Y^{\prime}$. Hence, we have the following inequalities (use (1.4.2)). 


$$
p_{a}\left(r Y_{\psi}\right) \geqq \frac{1}{2} r(\rho-2-r) \rho+1, \quad 0 \leqq r, \quad r: \text { integer. }
$$

The assertion follows from this.

\section{J. Dixon proves the following (Theorem 1 of [5]),}

Theorem 9. Let $(V, p)$ be a normal two-dimensional singularity of multiplicity two represented as $\left\{z^{2}-g(x, y)=0\right\}, o$ (cf. Introduction). If the multiplicity of the reduced analytic space $\{g(x, y)=0\}, o$ is even, the maximal ideal cycle coincides with the Artin's fundamental cycle, say $Z_{0}$, in all resolutions.

By this, the equality $p_{a}\left(Z_{0}\right)=\gamma_{1}$, in the condition of Theorem 9 above, follows from the equalities $(1.4 .4)$ and (1.4.6). Noting Lemma 1 , for any couple of integers $\left(\gamma_{1}, \beta\right)$ such that $\beta \geqq \gamma_{1} \geqq 2$, there is a singularity $(V, p)$ which satisfies the condition of Theorem 9 such that $p_{a}\left(Z_{0}\right)=\gamma_{1}, p_{a}(V, p) \geqq \beta$.

\section{References}

[1] Abhyanker, S. S., Local rings of high embedding dimension, Amer.J. Math., 89 (1967), 1073-1077.

[2] Artin, M., Some numerical criteria for contractability of curves on algebraic surfaces, Amer. J. Math., 84 (1962) 485-496.

[3] — On isolated rational singularity of surface, Amer.J. Math., 88 (1966), 129-136.

[4] Brieskorn, E., Über die Auflösung gewisser Singularitäten von holomorpher Abbildungen, Math. Ann., 166 (1966), 76-102.

[5] Dixon, D. J., The fundamental divisor of normal double points of surfaces, Pacific J. Math., 80 (1979), 105-115.

[6] Hartshorne, R. and Ogus, A., On the factoriality of local rings of small embedding codimension, Comm. Algebra, 1. (5) (1974), 415-437.

[7] Higuchi, T., Yoshinaga, E. and Watanabe, Kimio, Introduction to compler analysis of several variables, Morikita Library in Math., 51 (1980), (in Japanese).

[8] Horikawa, E., On deformations of quintic surfaces, Invent. Math., 31 (1975), 43-85.

[9] Kirby, D., The structure of an isolated multiple point of a surface I, II, III, Proc. London Math. Soc. (3), VI (1956), 597-609, VII (1957), 1-28.

[10] Laufer, H. B., Normal two-dimensional singularities, Ann. Math. Studies, 71 (1971).

[11] — On isolated rational singularities, Amer. J. Math., 94 (1972), 597-608.

[12] - On minimally elliptic singularities, Amer. J. Math., 99 (1977), 1257-1295.

[13] - On normal two-dimensional double point singularities, Israel J. Math., 31 (1978), 315-334.

[14] Lipman, J., Introductions to resolution of singularities, Proc. Symp. Pure. Math., 29 (1975), 187-230. 
[15] Samuel, P., Algebricite de certain points singuliers algebroids, J. Math. Pures. Appl., 35 (1956), 1-6.

[16] Siu, Y. T., Analytic sheaf cohomology groups of dimension $n$ of $n$-dimensional complex space, Trans. Amer. Math. Soc., 143 (1969), 77-94.

[17] Tjurina, G. N., Absolute isolatedness of rational singularities and triple points, Functional Anal. Appl., 2 (1968), 324-333.

[18] Wagreich, P., Elliptic singularities of surfaces, Amer. J. Math., 92 (1970), 419-454.

[19] Watanabe Kimio, On plurigenera of normal isolated singularities I, Math. Ann., 250 (1980), 65-94.

[20] Yau, S. S.-T., On maximally elliptic singularities, Trans. Amer. Math. Soc., 257 (1980).

[21] - Hypersurface weighted dual graphs of normal singularities of surfaces, Amer. J. Math., 101 (1979), 761-812.

[22] Zariski, O., The reduction of singularities of an algebraic surfaces, Ann. of Math., 40 (1939), 639-689. 